Open J. Math. Sci., Vol. 2(2018), No. 1, pp. 179 - 201

Website: https://pisrt.org/psr-press/journals/oms/

ISSN: 2523-0212 (Online) 2616-4906 (Print)

http://dx.doi.org/10.30538/oms2018.0027

\title{
FUZZY INTEGRAL DOMAINS AND FUZZY REGULAR SEQUENCES
}

\author{
SABA AYUB, WAQAS MAHMOOD ${ }^{1}$
}

\begin{abstract}
In this paper, the notions of fuzzy zero-divisors and fuzzy integral domains are illustrated. Some fundamental properties of fuzzy integral domains are proved. Moreover, the notions of fuzzy regular element and fuzzy regular sequences are defined. It is shown that any permutation (resp. any positive integral power) of a fuzzy regular sequence is again a fuzzy regular sequence. At the end, fuzzy regular sequences of two fuzzy submodules are related with the help of fuzzy short exact sequences.
\end{abstract}

Mathematics Subject Classification: 05C38, 15A15, 05A15, 15A18.

Key words and phrases: fuzzy zero-divisors; fuzzy integral domains; fuzzy regular sequences.

\section{Introduction}

The concept of fuzzy set theory was coined by L. Zadeh [1] to deal with the vagueness. This new theory provide a rich and meaningful improvement. Moreover, fuzzy set theory may be considered as a generalization of the classic set theory. Fuzzy sets has a wide range of applications in applied sciences such as computer sciences, management sciences, control sciences, robotics, artificial intelligence, pattern recognition and operation research etc. A. Rosenfeld, in [2], originated the concept of fuzzy groups and fuzzy subgroups. Since then many people have studied about fuzzy subgroups (see [3], [4], [5] and [6])

Most of the algebraic structures have been fuzzified by many researchers. Firstly, in [7], L. Wang-jin studied the fuzzy subrings and fuzzy ideals of a ring. L. W. Jin introduced some operations on fuzzy ideals (see [8]). Many other researchers

Received 02 February 2018. Revised 21 July 2018.

1 Corresponding Author

(C) 2018 Saba Ayub and Waqas Mahmood. This is an open access article distributed under the Creative Commons Attribution License, which permits unrestricted use, distribution, and reproduction in any medium, provided the original work is properly cited. 
continued the investigation of fuzzy subrings and fuzzy ideals (see [9], [10], [11] and [12]).

In [13], C.V. Negoita and D.A. Ralescu invented fuzzy submodules of a module. After that F. Pan defined fuzzy quotient modules, fuzzy finitely generated modules and fuzzy exact sequences (see [14], [15] and [16]). Moreover, he introduced

the notation of fuzzy homomorphism between fuzzy submodules. G. Muganda introduced the notation of free fuzzy modules (see [17]). In [18], R. Kumar, S. K. Bhambri, and P. Kumar presented some fundamental operations on fuzzy submodules.

This paper comprises six sections. Section 2 contains some auxiliary and preliminary results. In Section 3, fuzzy zero-divisors and fuzzy integral domains of an integral domain are defined. It is shown that the homomorphic image and preimage of a fuzzy integral domain is again a fuzzy integral domain. Also, some fundamental properties of a fuzzy integral domain are proved. Section 4 and 5 are devoted to the study of fuzzy regular elements and fuzzy regular sequences. Some results on fuzzy regular sequences are proved analogous to those that hold for the ordinary regular sequences. Finally, Section 6 consists of some concluded remarks.

\section{Preliminaries}

In this section, some basic definitions and fundamental results are given. In this paper, $R$ is denoted by a commutative ring with additive identity 0 and multiplicative identity 1 .

Definition 2.1. (see [1]) A fuzzy subset of a non-empty set $X$ is defined by a map

$$
\mu: X \rightarrow[0,1],
$$

also called a membership function which assigns degree of membership to each individual of $X$ in the unit interval $[0,1]$. The collection of all fuzzy subsets of a set $X$ is denoted by $F P(X)$.

$1_{R}$ is referred as the characteristic function of $R$. Let $\mu, \nu \in F P(R)$, then their intersection is defined as follows:

$$
(\mu \cap \nu)(x)=\mu(x) \wedge \nu(x),
$$

for all $x \in R$. Here, $\wedge$ denotes the infimum value.

Definition 2.2. ([19, Definition 1.1.3]) Let $a \in R$, and $0<\lambda \leq 1$. Then a fuzzy point, $a^{\lambda}$, of $R$ is a fuzzy subset which is defined as follows:

$$
a^{\lambda}(x)= \begin{cases}\lambda, & \text { if } x=a \\ 0, & \text { if } x \neq a\end{cases}
$$

for all $x \in R$. 
Definition 2.3. ([19, Definition 1.1.13]) Let $f: X \rightarrow Y$ be a function, $\mu \in$ $F P(X)$ and $\nu \in F P(Y)$. Then the fuzzy subset $f^{-1}(\nu)$ (resp. $\left.f(\mu)\right)$ of $X$ (resp. of $Y)$ is defined by:

$$
\begin{gathered}
f^{-1}(\nu)(x)=\nu(f(x)) \text { and } \\
f(\mu)(y)= \begin{cases}\vee\{\mu(a): a \in X, f(a)=y\}, & \text { if } f^{-1}(y) \neq \emptyset \\
0, & \text { otherwise }\end{cases}
\end{gathered}
$$

for all $x \in X$ and $y \in Y$. Here, $\vee$ denotes the supremum value.

Definition 2.4. ([7, Definition 3.1]) Let $\mu \in F P(R)$, then $\mu$ is called a fuzzy subring of $R$, if the following conditions hold:

(1) $\mu(r-s) \geq \mu(r) \wedge \mu(s)$,

(2) $\mu(r s) \geq \mu(r) \wedge \mu(s)$ and

(3) $\mu(0)=1$,

for all $r, s \in R$. The set of all fuzzy subrings of $R$ is denoted by $F S(R)$.

Definition 2.5. ([7, Definition 3.2]) Let $\mu \in F S(R)$, then it is called a fuzzy ideal of $R$, if $\mu(r s) \geq \mu(r) \vee \mu(s)$, for all $r, s \in R$.

Theorem 2.6. Let $\mu \in F S(R)$ and $I$ an ideal of $R$. Define $\xi \in F P(R / I)$ as follows:

$$
\xi(x+I)=\vee\{\mu(y): y+I=x+I\}
$$

for all $x \in R$. Then $\xi \in F S(R / I)$.

Proof. See [19, Theorem 3.2.10].

In the rest of paper, $\xi$ is called the quotient fuzzy subring of $R / I$ induced by $\mu$.

\section{Fuzzy Integral Domains}

In this section, fuzzy zero-divisors, fuzzy integral domains are defined and some significant results are proved.

Definition 3.1. Let $\mu \in F S(R)$, then a non-zero and non-unit element $r \in R$ is called a fuzzy zero-divisor over $\mu$, if there exists a non-zero and non-unit element $s \in R$ such that $\mu(r s)=1$. If a non-zero element is not a fuzzy zero-divisor over $\mu$, then it is called a fuzzy non-zero divisor over $\mu$.

Note that if $\mu \neq 0^{1}$ is a fuzzy sub-ring of $R$, then every zero-divisor over $R$ is a fuzzy zero-divisor over $\mu$. One can see that the converse is not true.

Definition 3.2. Let $R$ be an integral domain and $\mu \in F S(R)$. Then $\mu$ is called a fuzzy integral domain of $R$, if there does not exist any fuzzy zero-divisor over $\mu$. FID $(R)$ is denoted by the set of all fuzzy integral domains of $R$.

From now on, $R^{*}$ is denoted by the set of all unit elements of $R$. Moreover, in the rest of this section, $R$ is assumed to be an integral domain. Also, $\mu_{*}$ is denoting the following set:

$$
\mu_{*}=\{r \in R: \mu(r)=1\} .
$$


Example 3.3. Let $R=\mathbb{Q}[x]$ be the polynomial ring with rational coefficients and $\mu$ a fuzzy sub-ring of $R$ which is defined as:

$$
\mu(x)= \begin{cases}1, & \text { if } x \in \mathbb{Z} ; \\ 0.5, & \text { if } x \notin \mathbb{Z} .\end{cases}
$$

Here, $\mathbb{Z}$ denotes the ring of integers. Since $\mu_{*}=\mathbb{Z} \subseteq \mathbb{Q}=\{0\} \cup R^{*}$. By next Lemma 3.4 , it follows that $\mu$ is a fuzzy integral domain.

In the next result, a characterization of fuzzy integral domains is given.

Lemma 3.4. Let $\mu \in F S(R)$, then the following conditions are equivalent:

(1) $\mu \in F I D(R)$.

(2) $\mu(r) \neq 1$, for all non-zero and non-unit elements $r \in R$.

(3) $\mu_{*} \subseteq\{0\} \cup R^{*}$.

In particular, $0^{1}$ is a fuzzy integral domain of $R$.

Proof. Note that (2) and (3) are equivalent. We prove that (1) is equivalent to (2). Suppose that (2) holds and there exist $r, s \in R$ such that $\mu(r s)=1$. By (2), it follows that $r s=0$ or $r s$ is unit.

If $r s=0$, then either $r=0$ or $s=0$ since $R$ is an integral domain. Now, if $r s$ is unit, then both $r$ and $s$ are units. From each case we conclude that there does not exist any fuzzy zero-divisor over $\mu$. So, $\mu$ is a fuzzy integral domain of $R$.

Conversely, let $\mu \in F I D(R)$. Suppose that $\mu(r)=1$, for some non-zero element $r \in R$. Then, we have:

$$
\mu\left(r^{2}\right) \geq \mu(r) \wedge \mu(r)=1 .
$$

It implies that $\mu\left(r^{2}\right)=1$. If $r$ is non-unit, then $r$ will become a fuzzy zero-divisor over $\mu$ which is not possible. Hence, $r$ is unit. This proves that $\mu(r) \neq 1$, for all non-zero and non-unit elements $r \in R$.

Note that if $\mu$ is a fuzzy integral domain of $R$, then the equality $\mu_{*}=\{0\} \cup R^{*}$ is not true in general, see Example 3.3.

Remark 3.1. (i) Let $\mu \in F S(R)$, then the following conditions are equivalent:

(1) $R$ is a field.

(2) Every fuzzy sub-ring of $R$ is a fuzzy integral domain.

(3) $1_{R}$ is a fuzzy integral domain.

(ii) Let $\mu$ be a fuzzy ideal of $R$ such that $\mu \in F I D(R)$, then the following conditions are equivalent:

(1) $R / \mu=\{\mu\}$ i.e. $R / \mu$ is the zero ring.

(2) $r+\mu=0+\mu$, for all $r \in R$.

(3) $1+\mu=0+\mu$.

(4) $\mu(1)=1$.

(5) $\mu=1_{R}$.

Corollary 3.5. Let $\mu$ be a fuzzy ideal of $R$ such that $\mu \in F I D(R)$. Then the following hold: 
(1) If $\mu_{*} \neq\{0\}$, then $R$ is a field and $\mu=1_{R}$. In particular, $R / \mu=\{\mu\}$.

(2) If $R$ is not a filed, then $\mu_{*}=\{0\}$ and $\mu \neq 1_{R}$. In particular, $R / \mu$ is an integral domain.

Proof. (1) Suppose that $\mu_{*} \neq\{0\}$. By [19, Thoerem 3.1.10], $\mu_{*}$ is an ideal of $R$. Since $\mu$ is a fuzzy integral domain. By Lemma 3.4, we have $\mu_{*} \subseteq\{0\} \cup R^{*}$. Then there exists a unit element $r \in R$ such that $r \in \mu_{*}$. It follows that $\mu_{*}=R$. This proves that

$$
\mu=1_{R} \text { and } R=\{0\} \cup R^{*} .
$$

Hence, $R$ is a field. By Remark 3.1(ii), $R / \mu=\{\mu\}$.

(2) It is easy in view of (1) and Remark 3.1(i).

In the next Examples, it is shown that the assumptions in Corollary 3.5 are necessary.

Example 3.6. (1) Let $R=\mathbb{Z}[\sqrt{-5}]=\{a+b \sqrt{-5}: a, b \in \mathbb{Z}\}$ and $I=<$ $3,2+\sqrt{-5}>$. Let $\mu \in F P(R)$ such that

$\mu(x)= \begin{cases}1, & \text { if } x=0 ; \\ 1-\frac{1}{n_{x}}, & \text { where } n_{x} \text { is the smallest positive integer such that } x \notin I^{n_{x}} .\end{cases}$

Then $\mu$ is a fuzzy ideal of $R$ with $\mu_{*}=\{0\}$. By Lemma 3.4, $\mu$ is a fuzzy integral domain. Note that neither $R$ is a field nor $\mu=1_{R}$.

(2) Let $R=\mathbb{Z}[x]$. Suppose that $\mu \in F P(R)$ :

$$
\mu(r)= \begin{cases}1, & \text { if } r \in<x^{2}>\text { and } r \notin<x^{3}>; \\ 0, & \text { if } r \notin<x^{2}>; \\ 1-\frac{1}{4}, & \text { if } r \in<x^{3}>\text { and } r \notin<x^{4}>; \\ 1-\frac{1}{5}, & \text { if } r \in<x^{4}>\text { and } r \notin<x^{5}>; \\ \vdots & \vdots\end{cases}
$$

Then $\mu$ is a fuzzy ideal of $R$ with $\mu_{*}=<x^{2}>\nsubseteq R^{*} \cup\{0\}=\{0, \pm 1\}$. By Lemma $3.4, \mu$ is not a fuzzy integral domain. Note that neither $R$ is a field nor $\mu=1_{R}$. Moreover, $\left.R / \mu \cong R / \mu_{*}=\mathbb{Z}[x] /<x^{2}\right\rangle$ is neither the zero ring nor an integral domain.

Lemma 3.7. Let $\mu, \nu \in F S(R)$ such that $\mu \subseteq \nu$. If $\nu \in F I D(R)$, then $\mu \in$ $F I D(R)$.

Proof. By Lemma 3.4, it is obvious. Note that $\mu_{*} \subseteq \nu_{*}$ since $\mu \subseteq \nu$.

Corollary 3.8. Let $\mu \in F I D(R)$ and $\nu \in F S(R)$. Then $\mu \cap \nu \in F I D(R)$.

Proof. It is easy in view of Lemma 3.7 .

Suppose that $f: R \rightarrow S$ is the zero ring homomorphism between the integral domains such that $R$ is not a field. If $\nu \in F I D(S)$, then $f^{-1}(\nu)(r)=\nu(f(r))=$ $\nu(0)=1$, for all $r \in R$. In particular, $f^{-1}(\nu)=1_{R}$ which is not a fuzzy integral domain (see Remark 3.1(i)). Hence, under the zero ring homomorphism 
between the integral domains the pre-image of a fuzzy integral domain is not a fuzzy integral domain.

Now suppose that $\mu \in F S(R)$. Let $s \in S$ by any non-zero element. Since $f$ is the zero ring homomorphism, then by the definition of $f(\mu)$, we have:

$$
f(\mu)(s)=0, \text { for all non-zero elements } s \in S .
$$

This proves that $f(\mu)(s)=0^{1}$ a fuzzy integral domain of $S$ (see Lemma 3.4). Hence, under the zero ring homomorphism between the integral domains, the image of any fuzzy sub-ring is a fuzzy integral domain.

It seems to be unknown to the authors that the homomorphic image of any fuzzy sub-ring is also fuzzy sub-ring. So, we will prove it in the following Lemma 3.9. The similar result is true for fuzzy subgroups (see [20, Theorem 3.6]).

In the rest of this section, $0^{\prime}$ is denoted by the additive identity of the ring $S$.

Lemma 3.9. Let $f: R \rightarrow S$ be a ring homomorphism and $\mu \in F S(R)$. Then $f(\mu) \in F S(S)$.

Proof. Clearly, $f(\mu)\left(0^{\prime}\right)=1$. Now let $s_{1}, s_{2} \in S$ be any elements. If either $f^{-1}\left(\left\{s_{1}\right\}\right)=\emptyset$ or $f^{-1}\left(\left\{s_{2}\right\}\right)=\emptyset$, then by definition of $f(\mu)$, we have:

$$
f(\mu)\left(s_{1}\right)=0 \text { or } f(\mu)\left(s_{2}\right)=0 .
$$

It follows that in either case $f(\mu)\left(s_{1}\right) \wedge f(\mu)\left(s_{2}\right)=0$. Hence,

$$
f(\mu)\left(s_{1}+s_{2}\right) \geq f(\mu)\left(s_{1}\right) \wedge f(\mu)\left(s_{2}\right) \text { and } f(\mu)\left(s_{1} s_{2}\right) \geq f(\mu)\left(s_{1}\right) \wedge f(\mu)\left(s_{2}\right) .
$$

So, assume that $f^{-1}\left(\left\{s_{i}\right\}\right)$ is non-empty for each $i=1,2$. Then, by the proof of [19, Theorem 3.2.7], we have:

$$
f(\mu)\left(s_{1}+s_{2}\right) \geq f(\mu)\left(s_{1}\right) \wedge f(\mu)\left(s_{2}\right) \text { and } f(\mu)\left(s_{1} s_{2}\right) \geq f(\mu)\left(s_{1}\right) \wedge f(\mu)\left(s_{2}\right)
$$

From each case, we conclude that $f(\mu) \in F S(S)$.

Theorem 3.10. Let $R, S$ be two integral domains and $f: R \rightarrow S$ a non-zero ring homomorphism. Then the following are true:

(1) If $\mu \in F I D(R)$, then $f(\mu) \in F I D(S)$.

(2) If $\mu \in F I D(R)$, then $\mu$ is constant over $\operatorname{ker}(f)$ if and only if $f$ is injective.

(3) If $\nu \in F I D(S)$ such that $f^{-1}(\nu) \in F I D(R)$, then $f$ is injective.

(4) If $\nu \in F I D(S)$ and $f$ is injective such that $f\left(R^{*}\right)=S^{*}$. Then $f^{-1}(\nu) \in$ $F I D(R)$.

Proof. First of all note that $f(1)=1$ since $f: R \rightarrow S$ is a non-zero ring homomorphism between the integral domains. It follows that $f\left(R^{*}\right) \subseteq S^{*}$.

(1) Suppose that $\mu \in F I D(R)$. By Lemma 3.9, we have $f(\mu) \in F S(S)$. Now suppose that $s_{1} \in S$ such that $f(\mu)\left(s_{1}\right)=1$. Then

$$
1=f(\mu)\left(s_{1}\right)=\bigvee_{f(z)=s_{1}} \mu(z)
$$


It implies that $\mu(z)=1$, for some $z \in R$ such that $f(z)=s_{1}$. Since $\mu$ is a fuzzy integral domain of $R$, then by Lemma 3.4, it follows that $z=0$ or $z$ is a unit element. Hence, $s_{1}=0^{\prime}$ or $s_{1}$ is unit. It proves that $f(\mu)$ is a fuzzy integral domain of $S$, see Lemma 3.4.

(2) Suppose that $\mu \in F I D(R)$ and $\mu$ is constant over $\operatorname{ker}(f)$. Let $r \in \operatorname{ker}(f)$ be any element, then $f(r)=0^{\prime}=f(0)$. But $\mu$ is constant over $\operatorname{ker}(f)$, it follows that $\mu(r)=\mu(0)=1$. This proves that:

$$
r \in \mu_{*} \subseteq\{0\} \cup R^{*} \text {, see Lemma } 3.4 \text {. }
$$

But $f\left(R^{*}\right) \subseteq S^{*}$ and $f(r)=0^{\prime}$. Then it follows that $r=0$ and hence $\operatorname{ker}(f)=$ $\{0\}$. So, $f$ is injective. Note that the converse is obvious. Also, for the proof of (3), we refer to [19, Theorem 3.2.6] and (2).

(4) Let $\nu \in F I D(S)$ and $f$ be injective such that $f\left(R^{*}\right)=S^{*}$. Then it follows that $f^{-1}(\nu) \in F S(R)$ (see [19, Theorem 3.2.6]). Suppose that $f^{-1}(\nu)(r)=1$, where $r \in R \backslash\{0\}$. Since $f$ is injective, then $f(r) \in S \backslash\left\{0^{\prime}\right\}$. Then,

$$
\nu(f(r))=1 \text {. }
$$

It proves that $f(r) \in S^{*}$ since $\nu$ is a fuzzy integral domain of $S$ (see Lemma 3.4). But $f\left(R^{*}\right)=S^{*}$ and $f$ is injective, we conclude that $r$ is an element of $R^{*}$. Therefore, by Lemma $3.4, f^{-1}(\nu)$ is a fuzzy integral domain of $R$.

We now give Examples to show that if the assumption of Theorem 3.10(4) does not hold, then the pre-image of a fuzzy integral domain is not necessarily a fuzzy integral domain.

Example 3.11. (1) Let $R=\mathbb{Z}$ and $S=\mathbb{Q}$. Suppose that $f: R \rightarrow S$ is the natural inclusion map. Then $f\left(R^{*}\right)=\{ \pm 1\}$ is a proper subset of $S^{*}=\mathbb{Q} \backslash\{0\}$. Suppose that $\nu$ is a fuzzy subring of $S$ which is defined as:

$$
\nu(x)= \begin{cases}1, & \text { if } x \in \mathbb{Z} \\ 0.5, & \text { if } x \notin \mathbb{Z}\end{cases}
$$

Then $\nu_{*}=\mathbb{Z} \subseteq\{0\} \cup S^{*}$. So, we conclude that $\nu$ is a fuzzy integral domain of $S$, see Lemma 3.4. Note that $f^{-1}(\nu)(r)=\nu(f(r))=\nu(r)=1$, for all $r \in R$. By Remark 3.1(i), it follows that $f^{-1}(\nu)=1_{R}$ is not a fuzzy integral domain of $R$.

(2) Let $R=\mathbb{Q}[x], S=\mathbb{Q}[x] / I$ and $I=\langle x\rangle$, then it follows that:

$$
S^{*}=\{a+I: a \in \mathbb{Q} \backslash\{0\}\} \text { and } S \cong \mathbb{Q} .
$$

Let $f: R \rightarrow S$ be the natural projection map. Then note that $f\left(R^{*}\right)=S^{*}$ and $f$ is not injective.

Now, assume that $\nu=0^{1}$. Then it is a fuzzy integral domain of $S$. On the other hand, we have:

$$
f^{-1}(\nu)(r)=\nu(f(r))=\nu(r+I)= \begin{cases}1, & \text { if } r \in I \\ 0, & r \notin I\end{cases}
$$

Then $\left(f^{-1}(\nu)\right)_{*}=I \nsubseteq R^{*}$ and hence $f^{-1}(\nu)$ is not a fuzzy integral domain of $R$. 
Corollary 3.12. Let $R, S$ be two integral domains and $f: R \rightarrow S$ a non-zero ring homomorphism. Then the following conditions are equivalent:

(1) $f$ is injective.

(2) Every fuzzy integral domain of $R$ is constant over $\operatorname{ker}(f)$.

(3) $0^{1}$ is constant over $\operatorname{ker}(f)$.

Proof. It is easy in view of Theorem $3.10(2)$ and the fact that $0^{1}$ is a fuzzy integral domain of $R$.

Note that Example 3.11(2) shows that the claim in Corollary 3.12 is not true if $f$ is not injective. We will close this section with the following result related to fuzzy integral domains of quotient rings.

Proposition 3.13. Suppose that $\mu \in F I D(R)$ and $I$ is a prime ideal of $R$. Let $\xi$ be the quotient fuzzy subring of $R / I$ induced by $\mu$. Then $\xi \in F I D(R / I)$.

Proof. First of all note that $R / I$ is an integral domain. Let $\mu \in F I D(R)$, then by Theorem $2.6, \xi$ is the fuzzy sub-ring of $R / I$ induced by $\mu$.

Suppose that $\xi(r+I)=1$, for some $r \in R$. Then there exists $y \in r+I$ such that $\mu(y)=1$. Suppose that $y=r+a$ where $a \in I$. By Lemma 3.4, it follows that $r+a=0$ or $r+a$ is unit. Recall that $\mu$ is a fuzzy integral domain of $R$. If $r+a=0$ then $r \in I$. This proves that $r+I=I$. If $r+a$ is unit, then it follows that $r+I$ is unit. Hence, $\xi(r+I) \neq 1$, for all non-zero and non-unit elements $r+I \in R / I$. It implies that $\xi$ is a fuzzy integral domain of $R / I$ (see Lemma 3.4).

\section{Fuzzy Regular Sequences}

In this section, the notions of fuzzy regular elements and fuzzy regular sequences are given. Also, some interesting results concerning these ideas are proved. In the rest of paper, $M$ will be denoted as an $R$-module with additive identity $\theta$. First of all, we will define a regular element over $M$.

Definition 4.1. A non-zero element $r \in R$ is called an $M$-regular element, if $r m \neq \theta$, for all $m \in M \backslash\{\theta\}$.

Definition 4.2. ([19, Definition 4.1.8]) Let $\mu \in F P(M)$, then $\mu$ is called a fuzzy $R$-submodule of $M$, if the following conditions hold:

(1) $\mu(x-y) \geq \mu(x) \wedge \mu(y)$,

(2) $\mu(r x) \geq \mu(x)$ and

(3) $\mu(\theta)=1$,

for all $r \in R$ and $x, y \in M$. The set of all fuzzy $R$-submodules of $M$ will be denoted by $F S M(M)$.

Note that $\mu$ is a fuzzy ideal of $R$ if and only if $\mu \in F S M(R)$.

Definition 4.3. Let $\mu \in F S M(M)$. A non-zero element $r \in R$ is called a $\mu$-regular element(or fuzzy regular over $\mu$ ), if

$$
\mu(r m)=1 \text { for some } m \in M \Longrightarrow r m=\theta \text {. }
$$


Remark 4.1. (1) If $\mu_{*}=\{\theta\}$, then every non-zero element of $R$ is fuzzy regular over $\mu$.

(2) If $r \in R$ is both a $\mu$-regular and $M$-regular element, then $\mu_{*}=\{\theta\}$. Suppose that $x \in \mu_{*}$ then $\mu(x)=1$. It implies that $\mu(r x) \geq \mu(x)=1$ and hence $\mu(r x)=1$. But $r$ is $\mu$-regular, it follows that $r x=\theta$. Then $x=\theta$ since $r$ is $M$-regular. Hence, $\mu_{*}=\{\theta\}$.

(3) Converse of (2) is not true, see Example 4.4(2)(below).

(4) It is possible that there does not exist any fuzzy regular element over $\mu$, see Example 4.4(1)(below).

(5) In general, there is no relation between $\mu$-regular elements and $M$-regular elements, see Example 4.4(below).

(6) If $\mu$ is a fuzzy submodule of $M$ and $N$ a submodule of $M$. Suppose that $\left.\mu\right|_{N}$ is the restriction of $\mu$ over $N$. Then, it is obvious that $\left.\mu\right|_{N}$ is a fuzzy submodule of $N$. Moreover, every $\mu$-regular element is also a $\left.\mu\right|_{N}$-regular element.

Example 4.4. (1) Let $R=\mathbb{Z}_{4}$ and define a fuzzy ideal $\mu$ of $R$ as follows:

$$
\mu(x)= \begin{cases}1, & \text { if } x=\overline{0}, \overline{2} \\ 0, & \text { if } x=\overline{1}, \overline{3} .\end{cases}
$$

Note that $\mu(\overline{1} \cdot \overline{2})=\mu(\overline{2})=1$ and $\mu(\overline{2} \cdot \overline{3})=\mu(\overline{2})=1$. But $\overline{1} \cdot \overline{2} \neq \overline{0}$ and $\overline{2} \cdot \overline{3} \neq \overline{0}$. It follows that $\mu$ has no fuzzy regular element. Moreover, $\overline{1}$ is an $R$-regular element.

(2) Let $R=\mathbb{Z}, M=\mathbb{Z}_{4}$ and $\mu$ a fuzzy subset of $M$ which is defined as:

$$
\mu(x)= \begin{cases}1, & \text { if } x=\overline{0} \\ 0, & \text { if } x \neq \overline{0}\end{cases}
$$

Then $\mu \in F S M(M)$. Since $\mu_{*}=\{\overline{0}\}$, then every non-zero element of $R$ is fuzzy regular over $\mu$ (see Remark 4.1(1)). But $2 \cdot \overline{2}=\overline{0}$, it follows that $2 \in R$ is not an $M$-regular element.

Let $M_{1}$ be an $R$-submodule of $M$ and $\mu \in F S M(M)$. Suppose that $\xi$ is the fuzzy subset of the $R$-module $M / M_{1}$ :

$$
\xi(x+r M)=\sup \{\mu(y): y \in x+r M\}, \text { for all } x \in M .
$$

By [19, Theorem 4.2.1], $\xi \in F S M\left(M / M_{1}\right)$. From now on, $\xi$ is called the quotient fuzzy $R$-submodule of the $R$-module $M / M_{1}$ induced by $\mu$.

In the following result, the relation between fuzzy non-zero divisors and fuzzy regular elements is proved.

Lemma 4.5. Let $R$ be an integral domain and $\mu \neq 1_{R}$ a fuzzy ideal of $R$. If $r \in R$ is $\mu$-regular, then it is a fuzzy non-zero divisor over $\mu$. Converse is true if, $r$ is non-unit. Hence, a non-zero and non unit element of $R$ is $\mu$-regular if and only if it a fuzzy non-zero divisor over $\mu$.

Proof. Let $r \in R$ be $\mu$-regular, then $r \neq 0$. Assume that $\mu(r s)=1$, for some $s \in R$. By the assumption on $r$, it follows that $r s=0$ and hence $s=0$. This proves that $r$ is a fuzzy non-zero divisor over $\mu$. 
For the converse, let $r \in R \backslash R^{*} \cup\{0\}$ be a fuzzy non-zero divisor over $\mu$. Suppose that $\mu(r s)=1$, where $s \in R \backslash\{0\}$. Since $r$ is a fuzzy non-zero divisor over $\mu$, it implies that $s \in R^{*}$ and $r s \notin R^{*} \cup\{0\}$. Note that

$$
\mu((r s) r) \geq \mu(r s)=1 \text {. }
$$

This proves that $r$ is a fuzzy zero divisor over $\mu$ which is a contradiction. Hence, $s=0$. By definition, $r$ is $\mu$-regular.

Definition 4.6. Let $\mu \in F S M(M)$ and $r_{1}, \ldots, r_{n} \in R \backslash\{0\}$, then $r_{1}, \ldots, r_{n}$ is called a weak $\mu$-regular sequence (or weak fuzzy regular sequence over $\mu$ ), if the following conditions hold:

(1) $r_{1}$ is a $\mu$-regular,

(2) $r_{i}$ is a an $\xi_{i}$-regular element, for all $i \geq 2$.

Here, $\xi_{i}$ is the quotient fuzzy submodule of the $R$-module $M / \sum_{k=1}^{i-1} r_{k} M$ induced by $\mu$.

Note that there may exist an infinite weak fuzzy regular sequence. Let $R=\mathbb{Z}$, $x=2$ and $\mu$ is any fuzzy ideal of $R$ such that $x$ is fuzzy regular over $\mu$. Then we claim that $x, x^{2}, x^{3}, \ldots$ is an infinite weak $\mu$-regular sequence. Let $r_{i}=x^{i}$, for all $i \in \mathbb{N}$, then one can see that:

$$
\sum_{k=1}^{i} r_{k} R=\sum_{k=1}^{i-1} r_{k} R=2 R, \text { for all } i \geq 2 .
$$

It implies that $r_{i} \in \sum_{k=1}^{i} r_{k} R=\sum_{k=1}^{i-1} r_{k} R$, for all $i \geq 2$. Hence, $r_{i} x \in$ $\sum_{k=1}^{i-1} r_{k} M$, for all $x \in R$ and $i \geq 2$. This proves that:

$$
r_{i}\left(x+\sum_{k=1}^{i-1} r_{k} R\right)=r_{i} x+\sum_{k=1}^{i-1} r_{k} R=\sum_{k=1}^{i-1} r_{k} R,
$$

for all $x \in R$ and $i \geq 2$. Hence, $r_{i}$ is an $\xi_{i}$-regular element, for all $i \geq 2$. Here, $\xi_{i}$ is same as in Definition 4.6.

Definition 4.7. Let $\mu \in F S M(M)$ and $r_{1}, \ldots, r_{n} \in R \backslash\{0\}$, then $r_{1}, \ldots, r_{n}$ is called a $\mu$-regular sequence (or fuzzy regular sequence over $\mu$ ), if the following conditions hold:

(1) $r_{1}, \ldots, r_{n}$ is a weak $\mu$-regular sequence,

(2) For any $i \in\{2,3, \ldots, n\}$, we have:

$\sum_{k=1}^{i} r_{k} M \neq r_{1} M+r_{2} M+\cdots+r_{j-1} M+\widehat{r_{j} M}+r_{j+1} M+\cdots+r_{i} M$, for all $j \in 1,2, \ldots, i$, where $\widehat{r_{j} M}$ means that the term $r_{j} M$ does not appear.

(3) $M \neq \sum_{k=1}^{n} r_{k} M$. 
In the following Remark 4.2, it is shown that the length of any fuzzy regular sequence is finite. Note that $M$ is a Noetherian $R$-module, if every strictly increasing sequence of $R$-submodules of $M$ is stationary.

Remark 4.2. If $M$ is a Noetherian module over $R$ and $\mu \in F S M(M)$, then the length of any fuzzy regular sequence over $\mu$ is finite. Suppose on contrary that $r_{1}, r_{2}, \cdots \in R \backslash\{0\}$ is an infinite fuzzy regular sequence over $\mu$. By condition (2) in Definition 4.7, we have $\sum_{k=1}^{i} r_{k} M \neq \sum_{k=1}^{i-1} r_{k} M$, for all $i \geq 2$. It follows that the following sequence is a strictly increasing sequence of $R$-submodules of $M$ :

$$
r_{1} M \subset r_{1} M+r_{2} M \subset \cdots \subset \sum_{k=1}^{i-1} r_{k} M \subset \sum_{k=1}^{i} r_{k} M \subset \ldots
$$

which is a contradiction to the fact that $M$ is Noetherian.

Example 4.8. (1) If $R=\mathbb{Q}[x]$ and $\mu$ is a fuzzy ideal of $R$ such that $\mu_{*}=\{0\}$. Let $n \in \mathbb{N}$ be any fixed natural number and $r_{i}=2^{n+1-i}$, for all $1 \leq i \leq n$. Suppose that $\xi_{1}=\mu$ and $\xi_{i}$ is the quotient fuzzy submodule of the $R$-module $M / \sum_{k=1}^{i-1} r_{k} M$ induced by $\mu$, for all $2 \leq i \leq n$. Then we have:

$$
\left(\xi_{i}\right)_{*}=\{0\}, \text { for all } 2 \leq i \leq n .
$$

By Remark 4.1(1), it follows that $r_{i}$ is an $\xi_{i}$-regular element, for all $1 \leq i \leq n$. By Definition 4.6 , it follows that $r_{1}, \ldots, r_{n}$ is a weak $\mu$-regular sequence for any $n \in \mathbb{N}$. Note that for any $i \in\{2,3, \ldots, n\}$, the following equality hold:

$$
\sum_{k=1}^{i} r_{k} R=r_{i} R
$$

Hence, this proves that $r_{1}, \ldots, r_{n}$ is not a $\mu$-regular sequence for any $n \geq 2$.

(2) Let $R=\mathbb{Z}[x, y]$ denote the polynomial ring in two variables $x, y$ and $\mu \in F S M(R)$ such that $\mu_{*}=\{\theta\}$. Let $r_{1}=x, r_{2}=y$ and $r_{3}=p$, where $p$ is a fixed prime integer. Suppose that $N=r_{1} R+r_{2} R$ and $\xi_{2}$ (resp. $\xi_{3}$ ) is the quotient fuzzy submodule of the $R$-module $R / r_{1} R$ (resp. $R / N$ ) induced by $\mu$. Then we have:

$$
\left(\xi_{2}\right)_{*}=\left\{r_{1} R\right\} \text { and }\left(\xi_{3}\right)_{*}=\{N\} .
$$

By Remark 4.1(1), it follows that $r_{1}$ is $\mu$-regular, $r_{2}$ is $\xi_{2}$-regular and $r_{3}$ is $\xi_{3^{-}}$ regular. Note that the conditions (2) and (3) of Definition 4.7 also hold. Hence, $r_{1}, r_{2}, r_{3} \in R$ is a $\mu$-regular sequence.

We claim that $r_{1}, r_{2}, r_{3}$ is a $\mu$-regular sequence of maximal length. Let $I=<$ $r_{1}, r_{2}, r_{3}>$, then it is well-known that $I$ is a maximal ideal of $R$. Suppose that $r_{4} \in R$ is an arbitrary element. Then it follows that:

$$
<r_{1}, r_{2}, r_{3}, r_{4}>= \begin{cases}R, & \text { if } r_{4} \notin I \\ I, & \text { if } r_{4} \in I .\end{cases}
$$

From Definition 4.7, we conclude that $r_{1}, r_{2}, r_{3} \in R$ is a $\mu$-regular sequence of maximal length. This proves the claim. 
Proposition 4.9. Fix the notation of Definition 4.7. If $r_{1}, \ldots, r_{n} \in R \backslash\{0\}$ is a $\mu$-regular sequence. Then $r_{i}, \ldots, r_{n}$ is an $\xi_{i}$-regular sequence, for any $i \in$ $\{2,3, \ldots, n\}$.

Proof. Note that it suffices to prove the result for $i=2$ and $n=3$. By definition, $\xi_{2}$ is a quotient fuzzy submodule of the $R$-module $\bar{M}=M / r_{1} M$ induced by $\mu$. Denote the element $x+r_{1} M \in \bar{M}$ by $\bar{x}$.

Clearly, $r_{2}$ is $\xi_{2}$-regular. Let $\bar{N}=r_{2} \bar{M}$, then it is an $R$-submodule of $\bar{M}$. Let $\eta_{3}$ be the quotient fuzzy submodule of the $R$-module $\bar{M} / \bar{N}$ induced by $\xi_{2}$. We need to prove the following claims:

(i) $r_{3}$ is $\eta_{3}$-regular,

(ii) $r_{2} \bar{M}+r_{3} \bar{M} \neq r_{i} \bar{M}$, for all $i=2,3$, and

(iii) $\bar{M} \neq r_{2} \bar{M}+r_{3} \bar{M}$.

First of all, we show that $r_{2} \bar{M}+r_{3} \bar{M}$ is not equal to $r_{2} \bar{M}$. Suppose on contrary that $r_{2} \bar{M}+r_{3} \bar{M}=r_{2} \bar{M}$. Let $r_{3} x \in r_{3} M$ where $x \in M$. Then, we have:

$$
r_{3} x+r_{1} M=r_{3}\left(x+r_{1} M\right) \in r_{3} \bar{M} \subseteq r_{2} \bar{M}+r_{3} \bar{M}=r_{2} \bar{M} .
$$

It implies that $r_{3} x+r_{1} M=r_{2}\left(y_{2}+r_{1} M\right)=r_{2} y_{2}+r_{1} M$ with $y_{2} \in M$. Then we conclude that $r_{3} x-r_{2} y_{2} \in r_{1} M$ and hence

$$
r_{3} x=r_{2} y_{2}+r_{1} z \text { for some } z \in M .
$$

This proves that $r_{3} M \subseteq r_{1} M+r_{2} M$. So, we have $r_{1} M+r_{2} M+r_{3} M=r_{1} M+$ $r_{2} M$ which is a contradiction since $r_{1}, \ldots, r_{n}$ is a $\mu$-regular sequence. Hence, $r_{2} \bar{M}+r_{3} \bar{M} \neq r_{2} \bar{M}$. Similarly, one can prove that $r_{2} \bar{M}+r_{3} \bar{M} \neq r_{3} \bar{M}$ and $\bar{M} \neq r_{2} \bar{M}+r_{3} \bar{M}$. This completes the proof of the claims in (ii) and (iii).

Finally to prove the claim in $(i)$, let $\xi_{3}$ be the quotient fuzzy submodule of the $R$-module $M / M_{1}$ induced by $\mu$, where $M_{1}=r_{1} M+r_{2} M$. We firstly show that $\eta_{3}(\bar{x}+\bar{N}) \leq \xi_{3}\left(x+M_{1}\right)$, for all $x \in M$. Let $\bar{y} \in \bar{x}+\bar{N}$ be the element such that

$$
\eta_{3}(\bar{x}+\bar{N})=\xi_{2}(\bar{y}) .
$$

Note that $\bar{y} \in \bar{x}+\bar{N}$ if and only if

$$
\bar{y}=\bar{x}+r_{2} \bar{z}_{2}=x+r_{1} M+r_{2}\left(z_{2}+r_{1} M\right)=\left(x+r_{2} z_{2}\right)+r_{1} M
$$

with $z_{2} \in M$. It implies that $\xi_{2}(\bar{y})=\xi_{2}\left(\left(x+r_{2} z_{2}\right)+r_{1} M\right)$. So, let $u \in$ $\left(x+r_{2} z_{2}\right)+r_{1} M$ be the element such that

$$
\xi_{2}(\bar{y})=\xi_{2}\left(\left(x+r_{2} z_{2}\right)+r_{1} M\right)=\mu(u) .
$$

Note that we can write $u=\left(x+r_{2} z_{2}\right)+r_{1} z_{1} \in x+r_{1} M+r_{2} M=x+M_{1}$, where $z_{1} \in M$ and hence:

$$
\xi_{3}\left(x+M_{1}\right)=\sup \left\{\mu(t): t \in x+M_{1}\right\} \geq \mu(u) .
$$

From the Equations (1), (2) and (3), we conclude that $\eta_{3}(\bar{x}+\bar{N})$ is less than or equal to $\xi_{3}\left(x+M_{1}\right)$, for all $x \in M$. 
Now, we show that $r_{3}$ is $\eta_{3}$-regular. Suppose that $\eta_{3}\left(r_{3}(\bar{x}+\bar{N})\right)=\eta_{3}\left(\overline{r_{3} x}+\bar{N}\right)=$ 1 , for some $\bar{x} \in \bar{M}$. By the above remark, we have:

$$
\xi_{3}\left(r_{3}\left(x+M_{1}\right)\right)=\xi_{3}\left(r_{3} x+M_{1}\right)=1 .
$$

By the hypothesis, $r_{3}$ is $\xi_{3}$-regular. It follows that $r_{3} x+M_{1}=M_{1}$. Then we can write it as $r_{3} x=r_{1} w_{1}+r_{2} w_{2}$, where $w_{k} \in M$ for all $k=1,2$. Note that $r_{1} w_{1}+r_{1} M=r_{1} M$, then:

$$
\overline{r_{3} x}=r_{3} x+r_{1} M=r_{2} w_{2}+r_{1} M=r_{2} \bar{w}_{2} .
$$

Hence, $\overline{r_{3} x}+\bar{N}=\bar{N}$ and this shows that $r_{3}$ is $\eta_{3}$-regular. This completes the proof of Proposition.

It is well known that any positive power of a regular sequence over a module is again a regular sequence (see [21, Corollary 17.8]). We will prove that the similar result is true for fuzzy regular sequences.

Theorem 4.10. Let $\mu \in F S M(M)$ and $r_{1}, \ldots, r_{n} \in R \backslash\{0\}$ a $\mu$-regular sequence such that $r_{i}$ is a non-zero divisor over $R$, for all $i=1, \ldots, n$. Then $r_{1}^{a_{1}}, \ldots, r_{n}^{a_{n}}$ is also a $\mu$-regular sequence, where $a_{i}$ 's are any positive integers.

Proof. Firstly, we will prove by induction that $r_{1}^{a_{1}}$ is $\mu$-regular, for any positive integer $a_{1}$. Note that if $a_{1}=1$, then we are true. So let $a_{1}>1$, then note that $r_{1}^{b} \neq 0$, for any positive integer $b$ since $r_{1}$ is a non-zero divisor over $R$. Suppose that $\mu\left(r_{1}^{a_{1}} m\right)=1$ for some $\theta \neq m \in M$. Then it follows that $\mu\left(r_{1}^{a_{1}-1}\left(r_{1} m\right)\right)=1$. Then $r_{1} m=\theta$, since $r_{1}^{a_{1}-1} \neq 0$ and $r_{1}^{a_{1}-1}$ is $\mu$-regular. It follows that $r_{1}^{a_{1}} m=\theta$. So $r_{1}^{a_{1}}$ is $\mu$-regular for any positive integer $a_{1}$.

Next, we show that $r_{1}^{a_{1}}, r_{2}, \ldots, r_{n}$ is a $\mu$-regular sequence, for any positive integer $a_{1}$. Let $a_{1}=1$, then by the hypothesis we are true. Now assume that $a_{1}>1$ and $r_{1}^{a_{1}-1}, r_{2}, \ldots, r_{n}$ is a $\mu$-regular sequence. Note that $r_{1}^{a_{1}}$ is $\mu$-regular.

Let $i \in\{2, \ldots, n\}$ be fixed, $N:=r_{1}^{a_{1}} M+\sum_{k=2}^{i-1} r_{k} M$ and $\xi_{i}$ be the quotient fuzzy submodule of the $R$-module $M / N$ induced by $\mu$. To prove the result, we have to prove the following claims:

(i) $r_{i}$ is $\xi_{i}$-regular.

(ii) $r_{1}^{a_{1}} M+\sum_{k=2}^{i} r_{k} M \neq r_{1}^{a_{1}} M+r_{2} M+\cdots+r_{j-1} M+\widehat{r_{j} M}+r_{j+1} M+\cdots+$ $r_{i} M$, for all $j \in 1,2, \ldots, i$.

(iii) $M \neq r_{1}^{a_{1}} M+\sum_{k=1}^{n} r_{k} M$.

Note that it is enough to prove the claim in (ii) for $j=i$. Assume that $r_{1}^{a_{1}} M+\sum_{k=2}^{i} r_{k} M=N$, then $r_{i} M \subseteq N \subseteq r_{1}^{a_{1}-1} M+\sum_{k=2}^{i-1} r_{k} M$. It follows that $r_{1}^{a_{1}-1} M+\sum_{k=2}^{i} r_{k} M=r_{1}^{a_{1}-1} M+\sum_{k=2}^{i-1} r_{k} M$ which is not possible since $r_{1}^{a_{1}-1}, \ldots, r_{n}$ is a $\mu$-regular sequence. Hence, $N \neq r_{1}^{a_{1}} M+\sum_{k=2}^{i} r_{k} M$. This proves the claim in (ii). Similarly, we can prove the claim in $(i i i)$.

Now we show that the claim in $(i)$ is true. Suppose that $\xi_{i}\left(r_{i}(x+N)\right)=$ $\xi_{i}\left(r_{i} x+N\right)=1$, for some $x \in M$. By definition of $\xi_{i}$, there exists $y \in r_{i} x+N$ such that $\mu(y)=1$. 
Suppose that $T=r_{1}^{a_{1}-1} M+\sum_{k=2}^{i-1} r_{k} M$ and $\eta_{i}$ is the quotient fuzzy submodule of the $R$-module $M / T$ induced by $\mu$. Note that we can write:

$$
y=r_{i} x+r_{1}^{a_{1}-1}\left(r_{1} x_{1}\right)+\sum_{k=2}^{i-1} r_{k} x_{k},
$$

where $x_{k} \in M$, for all $k=1, \ldots, i-1$. It implies that $y+T=r_{i} x+T$. So, we have:

$$
\eta_{i}\left(r_{i}(x+T)\right)=\eta_{i}\left(r_{i} x+T\right)=\vee\left\{\mu(u): u \in r_{i} x+T\right\}=\mu(y)=1 .
$$

By induction hypothesis, the sequence $r_{1}^{a_{1}-1}, r_{2}, \ldots, r_{n}$ is a $\mu$-regular sequence. In particular, $r_{i}$ is $\eta_{i}$-regular, so we have $r_{i}(x+T)=T$. i.e. $r_{i} x \in T$. It implies that $r_{i} x=r_{1}^{a_{1}-1} w_{1}+\sum_{k=2}^{i-1} r_{k} w_{k}$ with $w_{k} \in M$, for all $k=1, \ldots, i-1$ and

$$
y=r_{i} x+r_{1}^{a_{1}} x_{1}+\sum_{k=2}^{i-1} r_{k} x_{k}=r_{1}^{a_{1}-1}\left(w_{1}+r_{1} x_{1}\right)+\sum_{k=2}^{i-1} r_{k}\left(x_{k}+w_{k}\right) .
$$

(see Equation (4)). Let $\eta_{i-1}$ be the quotient fuzzy submodule of the $R$-module $M / S$ induced by $\mu$, where $S=r_{1}^{a_{1}-1} M+\sum_{k=2}^{i-2} r_{k} M$. Then $y+S=r_{i-1}\left(x_{i-1}+\right.$ $\left.w_{i-1}\right)+S$. It implies that:

$$
\begin{aligned}
\eta_{i-1}\left(r_{i-1}\left(\left(x_{i-1}+w_{i-1}\right)+S\right)\right) & =\eta_{i-1}\left(r_{i-1}\left(x_{i-1}+w_{i-1}\right)+S\right) \\
& =\sup \left\{\mu(u): u \in r_{i-1}\left(x_{i-1}+w_{i-1}\right)+S\right\} \\
& =\mu(y)=1 .
\end{aligned}
$$

By induction, $r_{1}^{a_{1}-1}, r_{2}, \ldots, r_{n}$ is a $\mu$-regular sequence, so $r_{i-1}$ is $\eta_{i-1}$-regular. Then it follows that $r_{i-1}\left(x_{i-1}+w_{i-1}\right)+S=S$. Then, $r_{i-1}\left(x_{i-1}+w_{i-1}\right)=$ $r_{1}^{a_{1}-1} z_{1}+\sum_{k=2}^{i-2} r_{k} z_{k}$, where $z_{k} \in M$ for all $k=1, \ldots, i-2$. From Equation (5), we conclude that:

$y=r_{1}^{a_{1}-1}\left(w_{1}+r_{1} x_{1}\right)+\sum_{k=2}^{i-1} r_{k}\left(x_{k}+w_{k}\right)=r_{1}^{a_{1}-1}\left(r_{1} x_{1}+w_{1}+z_{1}\right)+\sum_{k=2}^{i-2} r_{k}\left(x_{k}+w_{k}+z_{k}\right)$.

Continuing in the same way, we can get that $y=r_{1}^{a_{1}-1}\left(r_{1} x_{1}+w_{1}+z_{1}+u_{1}+\right.$ $\left.\cdots+v_{1}\right)$, where $w_{1}, x_{1}, z_{1}, u_{1}, \ldots, v_{1} \in M$. Then,

$$
1=\mu(y)=\mu\left(r_{1}^{a_{1}-1}\left(r_{1} x_{1}+w_{1}+z_{1}+u_{1}+\cdots+v_{1}\right)\right) .
$$

Hence, $y=r_{1}^{a_{1}-1}\left(r_{1} x_{1}+w_{1}+z_{1}+u_{1}+\cdots+v_{1}\right)=\theta$ since $r_{1}^{a_{1}-1}$ is $\mu$-regular. It follows that $r_{i} x+N=y+N=N$. Hence, $r_{i}$ is $\xi_{i}$-regular. This proves the claim in $(i)$. Therefore, by induction, we conclude that $r_{1}^{a_{1}}, r_{2}, \ldots, r_{n}$ is a $\mu$-regular sequence for any positive integer $a_{1}$.

By Proposition 4.9, it follows that $r_{2}, \ldots, r_{n}$ is an $\xi_{2}$-regular sequence. By the above same arguments, we can prove that $r_{2}^{a_{2}}, \ldots, r_{n}$ is an $\xi_{2}$-regular sequence, for any positive integer $a_{2}$. Continuing in this way, we get that $r_{1}^{a_{1}}, \ldots, r_{n}^{a_{n}}$ is a $\mu$-regular sequence, where $a_{i}$ 's are any positive integers. 
If $R$ is a Noetherian local ring, then by [21, Corollary 17.2], every permutation of a regular sequence over a finitely generated $R$-module is again a regular sequence. Here, we are succeeded to prove this result in case of fuzzy regular sequences.

Theorem 4.11. If $r_{1}, \ldots, r_{n} \in R \backslash\{0\}$ is a $\mu$-regular sequence. Then any permutation of $r_{1}, \ldots, r_{n}$ is again a $\mu$-regular sequence.

Proof. Since every permutation can be written as a product of transpositions of adjacent elements. It suffices to prove that $r_{1}, \ldots, r_{i+1}, r_{i}, \ldots, r_{n}$ is a $\mu$-regular sequence. By Proposition 4.9, it is enough to prove the result for $i=1$.

Let $r_{1}, r_{2} \in R$ be a $\mu$-regular sequence. Let $\mu\left(r_{2} x\right)=1$, for some $x \in M$ and $\xi$ be the quotient fuzzy submodule of the $R$-module $M / r_{1} M$ induced by $\mu$.

By definition of $\xi$, it follows that $\xi\left(r_{2}\left(x+r_{1} M\right)\right)=\xi\left(r_{2} x+r_{1} M\right) \geq \mu\left(r_{2} x\right)=1$. Then we have $r_{2} x+r_{1} M=r_{1} M$ since $r_{2}$ is $\xi$-regular. Write $r_{2} x=r_{1} m$, for some $m \in M$. It implies that:

$$
\mu\left(r_{1} m\right)=\mu\left(r_{2} x\right)=1
$$

So, we have $r_{2} x=r_{1} m=\theta$ because of $r_{1}$ is $\mu$-regular. Hence, $r_{2}$ is $\mu$-regular. Note that $r_{1} R+r_{2} R \neq r_{i} R$, for all $i=1,2$. Now let $\eta$ be the quotient fuzzy submodule of the $R$-module $M / r_{2} M$ which is defined as:

$$
\eta\left(m+r_{2} M\right)=\sup \left\{\mu(y): y \in m+r_{2} M\right\}, \text { for all } m \in M .
$$

Let $\eta\left(r_{1}\left(x+r_{2} M\right)\right)=1$, where $x \in M$. It implies that $\mu(y)=1$ for some $y+r_{2} M=r_{1} x+r_{2} M$. Then $y=r_{1} x+r_{2} m$ with $m \in M$. Note that $y \in$ $r_{2}\left(m+r_{1} M\right)$, it follows that:

$$
\xi\left(r_{2}\left(m+r_{1} M\right)\right) \geq \mu(y)=1 .
$$

Since $r_{2}$ is $\xi$-regular, then we have $r_{2} m+r_{1} M=r_{1} M$. Let us write $r_{2} m=r_{1} z$ for some $z \in M$. Then,

$$
y=r_{1} x+r_{2} m=r_{1} x+r_{1} z=r_{1}(x+z) .
$$

It implies that $\mu\left(r_{1}(x+z)\right)=\mu(y)=1$. But $r_{1}$ is $\mu$-regular, so we have $y=$ $r_{1}(x+z)=\theta$. This proves that $r_{1} x+r_{2} M=r_{2} M$ and hence $r_{1}$ is $\eta$-regular. Therefore, $r_{2}, r_{1}$ is a $\mu$-regular sequence.

\section{Fuzzy homomorphisms and fuzzy exact sequences}

In this section, by $\mu_{X}$ we mean that a fuzzy subset of an $R$-module $X$. A fuzzy homomorphism $\widetilde{f}: \mu_{M} \rightarrow \nu_{N}$ between the fuzzy submodules is an $R$-module homomorphism $f: M \rightarrow N$ such that $\nu(f(x)) \geq \mu(x)$, for all $x \in M$ (see [14, Definition 1.1]). Moreover, $\widetilde{f}$ is called injective (resp. surjective), if $f$ is injective (resp. surjective). Also, $\operatorname{define} \operatorname{ker}(\widetilde{f}):=\left.\mu\right|_{\operatorname{ker}(f)}$ and $\operatorname{im}(\widetilde{f}):=\left.\nu\right|_{\operatorname{im}(f)}$.

Definition 5.1. ([22, Definition 3.1]) The sequence $\mu_{M} \stackrel{\widetilde{f}}{\rightarrow} \nu_{N} \stackrel{\widetilde{g}}{\rightarrow} v_{T}$ is called a fuzzy exact sequence of fuzzy submodules, if $\operatorname{im}(\widetilde{f})=\operatorname{ker}(\widetilde{g})$. 
It is clear, from the definition, that if $\mu_{M} \stackrel{\widetilde{f}}{\rightarrow} \nu_{N} \stackrel{\widetilde{g}}{\rightarrow} v_{T}$ is a fuzzy exact sequence, then the sequence $M \stackrel{f}{\rightarrow} N \stackrel{g}{\rightarrow} T$ is an exact sequence of $R$-modules, i.e. $\operatorname{im}(f)=$ $\operatorname{ker}(g)$.

Definition 5.2. ([22, Definition 3.1]) The sequence $\overline{1} \rightarrow \mu_{M} \stackrel{\widetilde{f}}{\rightarrow} \nu_{N} \stackrel{\widetilde{g}}{\rightarrow} v_{T} \rightarrow \overline{1}$ is called a fuzzy short exact sequence of fuzzy submodules, if $\widetilde{f}$ is injective, $\widetilde{g}$ is surjective and $\operatorname{im}(\widetilde{f})=\operatorname{ker}(\widetilde{g})$. In particular, the sequence $0 \rightarrow M \stackrel{f}{\rightarrow} N \stackrel{g}{\rightarrow} T \rightarrow 0$ is a short exact sequence of $R$-modules.

Remark 5.1. If $0 \rightarrow M \stackrel{f}{\rightarrow} N \stackrel{g}{\rightarrow} T \rightarrow 0$ is a short exact sequence of $R$-modules such that $\mu_{M} \stackrel{\widetilde{f}}{\rightarrow} \nu_{N}$ and $\nu_{N} \stackrel{\widetilde{g}}{\rightarrow} v_{T}$ are fuzzy homomorphisms. Then the sequence $\overline{1} \rightarrow \mu_{M} \stackrel{\widetilde{f}}{\rightarrow} \nu_{N} \stackrel{\widetilde{g}}{\rightarrow} v_{T} \rightarrow \overline{1}$ is a fuzzy short exact sequence.

Lemma 5.3. Let $\tilde{f}: \mu_{M} \rightarrow \nu_{N}$ be a fuzzy homomorphism between fuzzy submodules. Suppose that $M_{1}$ and $N_{1}$ are submodules of $M$ and $N$ respectively such that $f\left(M_{1}\right) \subseteq N_{1}$. Let $\xi$ and $\gamma$ be the quotient fuzzy submodules of the $R$-modules $M / M_{1}$ and $N / N_{1}$ induced by $\mu_{M}$ and $\nu_{N}$ respectively. Then $\tilde{f}$ induces a fuzzy homomorphism:

$$
\tilde{f}_{1}: \xi \rightarrow \gamma, m+M_{1} \mapsto f(m)+N_{1}
$$

for all $m \in M$.

Proof. Suppose that $\xi$ and $\gamma$ are the quotient fuzzy submodules of the $R$-modules $M / M_{1}$ and $N / N_{1}$ induced by $\mu_{M}$ and $\nu_{N}$ respectively. Since $f$ is an $R$-module homomorphism and $f\left(M_{1}\right) \subseteq N_{1}$, then $f$ induces the following $R$-module homomorphism:

$$
f_{1}: M / M_{1} \rightarrow N / N_{1}, m+M_{1} \mapsto f(m)+N_{1},
$$

for all $m \in M$. We only need to show that $\gamma\left(f_{1}\left(x+M_{1}\right)\right) \geq \xi\left(x+M_{1}\right)$, for all $x+M_{1} \in M / M_{1}$. Let $x+M_{1} \in M / M_{1}$ be an arbitrary element. Suppose that $m \in M$ such that:

$$
m \in x+M_{1} \text { and } \xi\left(x+M_{1}\right)=\mu(m) .
$$

Then $m+M_{1}=x+M_{1}$ and it follows that $f(m)+N_{1}=f(x)+N_{1}$. Since $\tilde{f}: \mu_{M} \rightarrow \nu_{N}$ is a fuzzy $R$-module homomorphism. Then by Equation (6), we have:

$$
\gamma\left(f_{1}\left(x+M_{1}\right)\right)=\gamma\left(f(x)+N_{1}\right) \geq \nu(f(m)) \geq \mu(m)=\xi\left(x+M_{1}\right) .
$$

This proves that $\tilde{f}_{1}: \xi \rightarrow \gamma$ is a fuzzy homomorphism.

Corollary 5.4. Let $\mu_{M} \stackrel{\widetilde{f}}{\rightarrow} \nu_{N} \stackrel{\widetilde{g}}{\rightarrow} v_{T} \rightarrow \overline{1}$ be a fuzzy exact sequence of fuzzy submodules. Suppose that $M_{1}, N_{1}$ and $T_{1}$ are submodules of $M, N$ and $T$ respectively such that $f\left(M_{1}\right) \subseteq N_{1}$ and $g\left(N_{1}\right) \subseteq T_{1}$. Let $\xi, \gamma$ and $\eta$ be the quotient fuzzy submodules of the R-modules $M / M_{1}, N / N_{1}$ and $T / T_{1}$ induced by $\mu_{M}, \nu_{N}$ 
and $v_{T}$ respectively. Then the following sequence is a fuzzy exact sequence of fuzzy submodules:

$$
\xi \stackrel{\widetilde{f_{1}}}{\rightarrow} \gamma \stackrel{\widetilde{g_{1}}}{\rightarrow} \eta \rightarrow \overline{1} .
$$

Here, $f_{1}$ and $g_{1}$ are the maps induced by $f$ and $g$ respectively. In addition, if $f$ is injective and $\gamma\left(x+N_{1}\right) \neq 1$, for all non-zero elements $x \in \operatorname{im}(f)$. Then $f_{1}$ is injective.

Proof. Since $f\left(M_{1}\right) \subseteq N_{1}$ and $g\left(N_{1}\right) \subseteq T_{1}$. By Lemma 5.3, the maps $\widetilde{f}_{1}: \xi \rightarrow \gamma$ and $\widetilde{g_{1}}: \gamma \rightarrow \eta$ are the fuzzy homomorphisms induced by $f$ and $g$ respectively. Moreover, the sequence $M \stackrel{f}{\rightarrow} N \stackrel{g}{\rightarrow} T \rightarrow 0$ is an exact sequence of $R$-modules. It is well-known that, it induces the following exact sequence of $R$-modules:

$$
M / M_{1} \stackrel{f_{1}}{\rightarrow} N / N_{1} \stackrel{g_{1}}{\rightarrow} T / T_{1} \rightarrow 0 .
$$

Hence, the result follows from Remark 5.1.

Now, we prove the injectivity of $f_{1}$. Suppose that $f$ is injective and $\gamma\left(x+N_{1}\right) \neq 1$, for all non-zero elements $x \in \operatorname{im}(f)$. Let $f_{1}\left(m+M_{1}\right)=f(m)+N_{1}=N_{1}$ for some $m \in M$. Then we have $\gamma\left(f(m)+N_{1}\right)=1$. It implies that $f(m)=\theta$ and hence $m=\theta$. This proves that $m+M_{1}=M_{1}$ and hence $\operatorname{ker}\left(f_{1}\right)$ is zero.

Lemma 5.5. Let $\tilde{f}: \mu_{M} \rightarrow \nu_{N}$ be a fuzzy homomorphism of fuzzy submodules. Suppose that the following conditions hold:

(i) $r_{1} \in R \backslash\{0\}$ is fuzzy regular over $\nu_{N}$ and

(ii) $\mu(x) \neq 1$, for all non-zero elements $x \in \operatorname{ker}(f)$.

Then $r_{1}$ is also fuzzy regular over $\mu_{M}$. In particular, if $f$ is injective, then every $\nu$-regular element is also a $\mu$-regular element.

Proof. Suppose that $\mu\left(r_{1} x\right)=1$, for some $x \in M$. We claim that $r_{1} x \in \operatorname{ker}(f)$. Since $\nu\left(r_{1} f(x)\right)=\nu\left(f\left(r_{1} x\right)\right) \geq \mu\left(r_{1} x\right)=1$. It implies that $\nu\left(r_{1} f(x)\right)=1$. By the assumption in $(i)$, we have:

$$
f\left(r_{1} x\right)=r_{1} f(x)=\theta .
$$

It follows that $r_{1} x \in \operatorname{ker}(f)$. This completes the proof of the claim. Hence, by the assumption in $(i i)$, we conclude that $r_{1} x=\theta$. Recall that $\mu\left(r_{1} x\right)=1$. This proves that $r_{1}$ is $\mu$-regular.

In the next Example, we will prove that the assumption of Lemma 5.5(ii) is necessary. Moreover, if $\widetilde{f}: \mu_{M} \rightarrow \nu_{N}$ is an onto fuzzy homomorphism of fuzzy submodules. Then a $\nu$-regular element is not necessarily a $\mu$-regular element. Also, in Example 5.8(below), it is shown that a $\mu$-regular element is not necessarily a $\nu$-regular element even $f$ is injective.

Example 5.6. Let $R=\mathbb{Z}, N=\mathbb{Z}_{2}$ and $M=\mathbb{Z}_{2} \times \mathbb{Z}_{2}$. Then $M \stackrel{f}{\rightarrow} N$ is an onto $R$-module homomorphism with $f(s, r)=s$, for all $r, s \in N$. Define fuzzy 
submodules $\mu_{M}$ and $\nu_{N}$ as:

$$
\begin{gathered}
\mu(r)= \begin{cases}1, & \text { if } r=(\overline{0}, \overline{0}) \text { or }(\overline{0}, \overline{1}) ; \\
0.1, & \text { if } r=(\overline{1}, \overline{0}) \text { or }(\overline{1}, \overline{1}) .\end{cases} \\
\text { and } \nu(r)= \begin{cases}1, & \text { if } r=\overline{0} ; \\
0.1, & \text { if } r=\overline{1} .\end{cases}
\end{gathered}
$$

for all $r \in N$. Now, we show that $\widetilde{f}$ is a fuzzy homomorphism. To do this note that:

$$
\nu\left(f\left(\left(r_{1}, r_{2}\right)\right)\right)=\nu\left(r_{1}\right)= \begin{cases}1=\mu\left(\left(r_{1}, r_{2}\right)\right), & \text { if } r_{1}=\overline{0} \\ 0.1=\mu\left(\left(r_{1}, r_{2}\right)\right), & \text { if } r_{1}=\overline{1}\end{cases}
$$

for all $r_{1}, r_{2} \in N$. This proves that $\widetilde{f}$ is a fuzzy homomorphism. Since $\nu_{*}=\{0\}$, then every non-zero integer is a $\nu$-regular element (see Remark 4.1(1)).

Note that $(\overline{0}, \overline{1}) \in \operatorname{ker}(f)$ is non-zero and $\mu((\overline{0}, \overline{1}))=1$. Hence, the assumption of $(i i)$ in Lemma 5.5 does not hold.

Now let $r_{1}=3$ and $m=(\overline{0}, \overline{1}) \in M$, then $r_{1} m \neq(\overline{0}, \overline{0})$ and $\mu\left(r_{1} m\right)=1$. This proves that $r_{1}=3$ is not a fuzzy regular over $\mu$. One can see that every odd integer is not a fuzzy regular over $\mu$.

Corollary 5.7. Let $M_{1}$ be a submodule of $M$. Let $\mu \in F S M(M)$ and $\gamma$ be the quotient fuzzy submodule of the $R$-module $M / M_{1}$ induced by $\mu$. Suppose that the following conditions hold:

(i) $r_{1} \in R \backslash\{0\}$ is fuzzy regular over $\gamma$.

(ii) $\mu(x) \neq 1$, for all non-zero elements $x \in M_{1}$.

Then $r_{1}$ is also fuzzy regular over $\mu$.

Proof. First of all note that there is an $R$-module homomorphism:

$$
M \stackrel{\pi}{\rightarrow} M / M_{1}, x \mapsto x+M_{1},
$$

for all $x \in M$. Since $\gamma(\pi(x))=\gamma\left(x+M_{1}\right) \geq \mu(x)$, for all $x \in M$. This proves that $\tilde{\pi}: \mu \rightarrow \gamma$ is a fuzzy homomorphism. Note that by assumption in (ii), we have $\mu(x) \neq 1$, for all non-zero elements $x \in M_{1}=\operatorname{ker}(\pi)$. Then the result follows from Lemma 5.5.

Let $\tilde{f}: \mu_{M} \rightarrow \nu_{N}$ be a fuzzy homomorphism of fuzzy submodules. Suppose that $\gamma$ is the quotient fuzzy submodule of the $R$-module $\operatorname{coker}(f):=N / \operatorname{im}(f)$ induced by $\nu_{N}$ and $\nu(x) \neq 1$, for all non-zero elements $x \in \operatorname{im}(f)$. By Corollary 5.7 (for $N_{1}=\operatorname{im}(f)$ ), every fuzzy regular element over $\gamma$ is also a fuzzy regular element over $\nu_{N}$.

It is well know that if $0 \rightarrow M \stackrel{f}{\rightarrow} N \stackrel{g}{\rightarrow} T \rightarrow 0$ is an exact sequence of $R$-modules such that $r_{1}, \ldots, r_{n} \in R \backslash\{0\}$ is a regular sequence over $M$ and $T$. Then it is also a regular sequence over $N$. In the next Example, we will prove that this result is not true in case of fuzzy submodules. 
Example 5.8. Let $R=\mathbb{Z}, M=\mathbb{Z}_{2}, N=\mathbb{Z}_{2} \times \mathbb{Z}_{2}$ and $M \stackrel{f}{\rightarrow} N$ be the morphism with $f(u)=(0, u)$, for all $u \in M$. Then $f$ is an injective $R$-module homomorphism. Suppose that $T=\operatorname{coker}(f)=\{\operatorname{im}(f),(\overline{1}, \overline{0})+\operatorname{im}(f)\}$. Then the following sequence is exact:

$$
0 \rightarrow M \stackrel{f}{\rightarrow} N \stackrel{g}{\rightarrow} T \rightarrow 0,
$$

where $g((u, w))=(u, w)+\operatorname{im}(f)$, for all $u, w \in M$. Define $\mu_{M}, \nu_{N}$ and $v_{T}$ as:

$$
\begin{gathered}
\mu(r)= \begin{cases}1, & \text { if } r=\overline{0} ; \\
0.1, & \text { if } r=\overline{1} .\end{cases} \\
\nu((u, w))= \begin{cases}1, & \text { if }(u, w)=(\overline{0}, \overline{0}) \text { or }(\overline{0}, \overline{1}) ; \\
0.1, & \text { if }(u, w)=(\overline{1}, \overline{0}) \text { or }(\overline{1}, \overline{1}) .\end{cases} \\
v((u, w)+\operatorname{im}(f))=\vee\{\nu((y, z)):(y, z)+\operatorname{im}(f)=(u, w)+\operatorname{im}(f)\},
\end{gathered}
$$

for all $r, u, w \in M$. Clearly, $\mu$ and $\nu$ are fuzzy submodules of $M$ and $N$ respectively. It implies that $v_{T}$ is a fuzzy submodule of $T$ (see [19, Theorem 4.2.1]). By Example 5.6, it follows that $\widetilde{f}$ is a fuzzy homomorphism. Also, by definition of $v_{T}, \widetilde{g}$ is a fuzzy homomorphism. By Remark 5.1, the following sequence is a fuzzy short exact sequence of fuzzy submodules:

$$
\overline{1} \rightarrow \mu_{M} \stackrel{\widetilde{f}}{\rightarrow} \nu_{N} \stackrel{\widetilde{g}}{\rightarrow} v_{T} \rightarrow \overline{1}
$$

On the other hand ,we have:

$$
v((\overline{1}, \overline{0})+\operatorname{im}(f))=\nu((\overline{1}, \overline{0})) \vee \nu((\overline{1}, \overline{1}))=0.1 \neq 1
$$

This proves that $v_{*}=\{\operatorname{im}(f)\}$ and hence every non-zero integer is an $v$-regular element (see Remark 4.1(1)). From Example 5.6, every non-zero integer is a $\mu$-regular element and every odd integer is not a fuzzy regular over $\nu$.

Note that if $\overline{1} \rightarrow \mu_{M} \stackrel{\widetilde{f}}{\rightarrow} \nu_{N} \stackrel{\widetilde{g}}{\rightarrow} v_{T} \rightarrow \overline{1}$ is a fuzzy short exact sequence of fuzzy submodules. By Lemma 5.5, every $\nu_{N}$-regular element is also $\mu_{M}$-regular element. But in the next Example, it is shown that a $\nu_{N}$-regular element is not necessarily an $v_{T}$-regular element.

Example 5.9. Let $R=\mathbb{Z}[x], N=R \times R$ and $R \stackrel{f}{\rightarrow} N$ be the morphism with $f(u)=(0, u)$, for all $u \in R$. Let $g: N \rightarrow R$ be defined as $g((u, w))=u$, for all $u, w \in R$. Then the following sequence is exact:

$$
0 \rightarrow R \stackrel{f}{\rightarrow} N \stackrel{g}{\rightarrow} R \rightarrow 0 .
$$

Let us define the fuzzy submodules $\mu_{R}, \nu_{N}$ and $v_{R}$ as follows:

$$
\begin{gathered}
\mu(u)= \begin{cases}1, & \text { if } u=0 ; \\
0.5, & \text { if } u=0 .\end{cases} \\
\nu((u, w))= \begin{cases}1, & \text { if }(u, w)=(0,0) ; \\
0.5, & \text { if }(u, w) \neq(0,0) .\end{cases}
\end{gathered}
$$




$$
v(u)= \begin{cases}1, & \text { if } u \in<x> \\ 0.5, & \text { if } u \notin<x>\end{cases}
$$

where $u, w \in R$. By following the same lines as in proof of Example 5.6, it can be proved that $\widetilde{f}$ and $\widetilde{g}$ are fuzzy homomorphisms. Then the following sequence is a fuzzy short exact sequence of fuzzy submodules:

$$
\overline{1} \rightarrow \mu_{R} \stackrel{\widetilde{f}}{\rightarrow} \nu_{N} \stackrel{\widetilde{g}}{\rightarrow} v_{R} \rightarrow \overline{1}
$$

Let $r \in R \backslash\{0\}$ be an arbitrary element. Since $\mu_{*}=\{0\}$ and $\nu_{*}=\{(0,0)\}$, then it follows that $r$ is fuzzy regular over $\mu_{R}$ and $\nu_{N}$ (see Remark 4.1(1)).

Since $\langle x\rangle$ is an ideal of $R$. Then $r u \in\langle x\rangle$, for all $u \in\langle x\rangle$. It implies that $v(r u)=1$, for all $u \in<x>\backslash\{0\}$. Also, we have $r u \neq 0$, for all $u \in<x>\backslash\{0\}$ since $R$ is an integral domain. Hence, $r$ is not fuzzy regular over $v_{R}$, for all $r \in R \backslash\{0\}$.

Theorem 5.10. Let $\widetilde{g}: \nu_{N} \rightarrow v_{T}$ be an onto fuzzy homomorphism between fuzzy submodules. Suppose that $r_{1}, r_{2} \in R \backslash\{0\}$ satisfy the following conditions:

(i) $r_{1}, r_{2}$ is a fuzzy regular sequence over $v_{T}$.

(ii) If $N_{2}=r_{1} N$ and $\gamma_{2}$ is the quotient fuzzy submodule of the R-module $N / N_{2}$ induced by $\nu_{N}$. Assume that $\gamma_{2}\left(x+N_{2}\right) \neq 1$, for all non-zero elements $x \in \operatorname{ker}(g)$.

Then $r_{1}, r_{2}$ is also a fuzzy regular sequence over $\nu_{N}$.

Proof. First of all, we show that $\nu(x) \neq 1$, for all non-zero elements $x \in \operatorname{ker}(g)$. Suppose that $\nu(x)=1$, for some $x \in \operatorname{ker}(g)$. Then it implies that

$$
\gamma_{2}\left(x+N_{2}\right) \geq \nu(x)=1 .
$$

By assumption in (ii), we have $x=\theta$. Then $r_{1}$ is $\nu_{N}$-regular (see Lemma 5.5). Now we show that $r_{1} N \neq r_{1} N+r_{2} N$. Suppose that $r_{1} N=r_{1} N+r_{2} N$, then we have $r_{2} N \subseteq r_{1} N$. Since $g$ is onto. Then $g(N)=T$ and it follows that:

$$
r_{2} T=r_{2} g(N)=g\left(r_{2} N\right) \subseteq g\left(r_{1} N\right)=r_{1} g(N)=r_{1} T .
$$

Hence, we have $r_{1} T=r_{1} T+r_{2} T$ which is a contradiction since $r_{1}, r_{2}$ is a regular sequence over $v_{T}$. So, we conclude that $r_{1} N \neq r_{1} N+r_{2} N$. Similarly, we can prove that:

$$
r_{2} N \neq r_{1} N+r_{2} N \text { and } N \neq r_{1} N+r_{2} N \text {. }
$$

Finally, we prove that $r_{2}$ is $\gamma_{2}$-regular. Assume that $\gamma_{2}\left(r_{2} x+N_{2}\right)=1$, for some $x \in N$. Let us fix the following notations:

$$
M_{2}=r_{1} M, T_{2}=r_{1} T \text { and } \mu_{M}=\left.\nu\right|_{M},
$$

where $M=\operatorname{ker}(g)$ and $\left.\nu\right|_{M}$ is the restriction of $\nu$ over $M$. Suppose that $\xi_{2}$ and $\eta_{2}$ are the quotient fuzzy submodules of the $R$-modules $M / M_{2}$ and $T / T_{2}$ induced by $\mu_{M}$ and $v_{T}$ respectively.

Now, let $f: M \rightarrow N$ be the inclusion map. Then the sequence $M \stackrel{f}{\rightarrow} N \stackrel{g}{\rightarrow} T \rightarrow 0$ is an exact sequence of $R$-modules and $\widetilde{f}: \mu_{M} \rightarrow \nu_{N}$ is a fuzzy homomorphism. 
By Remark 5.1, it follows that the following sequence is a fuzzy exact sequence of fuzzy submodules:

$$
\mu_{M} \stackrel{\widetilde{f}}{\rightarrow} \nu_{N} \stackrel{\widetilde{g}}{\rightarrow} v_{T} \rightarrow \overline{1}
$$

Note that $f\left(M_{2}\right) \subseteq N_{2}$ and $g\left(N_{2}\right) \subseteq T_{2}$. By Corollary 5.4, it induces the following fuzzy exact sequence of fuzzy submodules:

$$
\xi_{2} \stackrel{\widetilde{f_{1}}}{\rightarrow} \gamma_{2} \stackrel{\widetilde{g_{1}}}{\rightarrow} \eta_{2} \rightarrow \overline{1}
$$

where $f_{1}\left(m+M_{2}\right)=f(m)+N_{2}=m+M_{2}$ and $g_{1}\left(a+N_{2}\right)=g(a)+T_{2}$, for all $m \in M$ and $a \in N$. Note that:

$$
\eta_{2}\left(r_{2} g_{1}\left(x+N_{2}\right)\right)=\eta_{2}\left(g_{1}\left(r_{2} x+N_{2}\right)\right) \geq \gamma_{2}\left(r_{2} x+N_{2}\right)=1
$$

But $r_{2}$ is $\eta_{2}$-regular, it follows that $g_{1}\left(r_{2} x+N_{2}\right)=r_{2} g_{1}\left(x+N_{2}\right)=T_{2}$. Then $r_{2} x+N_{2} \in \operatorname{ker}\left(g_{1}\right)=\operatorname{im}\left(f_{1}\right)$. There exists $m+M_{2} \in M / M_{2}$ such that:

$f_{1}\left(m+M_{2}\right)=m+N_{2}=r_{2} x+N_{2}$ and $\gamma_{2}\left(f_{1}\left(m+M_{2}\right)\right)=\gamma_{2}\left(m+N_{2}\right)=\gamma_{2}\left(r_{2}\left(x+N_{2}\right)\right)=1$

Since $m \in \operatorname{im}(f)=\operatorname{ker}(g)$, then by the assumption in (ii), we have $m=\theta$. Hence, $r_{2} x+N_{2}=N_{2}$. This proves that $r_{2}$ is $\gamma_{2}$-regular. This completes the proof of Theorem.

In the following Corollary, we will generalize the result of Theorem 5.10.

Corollary 5.11. Let $\widetilde{g}: \nu_{N} \rightarrow v_{T}$ be an onto fuzzy homomorphism between fuzzy submodules. Suppose that $r_{1}, \ldots, r_{n} \in R \backslash\{0\}$ satisfy the following conditions:

(i) $r_{1}, \ldots, r_{n}$ is a fuzzy regular sequence over $v_{T}$ and

(ii) If $j \in\{2, \ldots, \ldots n\}$ is fixed, $N_{j}=\sum_{k=1}^{j-1} r_{k} N$ and $\gamma_{j}$ is the quotient fuzzy submodule of the $R$-module $N / N_{j}$ induced by $\nu_{N}$. Assume that $\gamma_{j}\left(x+N_{j}\right) \neq 1$, for all non-zero elements $x \in \operatorname{ker}(g)$.

Then, $r_{1}, \ldots, r_{j}$ is also a fuzzy regular sequence over $\nu_{N}$.

Proof. Suppose that $j \in\{2, \ldots, \ldots n\}$ is fixed, $N_{j}=\sum_{k=1}^{j-1} r_{k} N$ and $\gamma_{j}$ is the quotient fuzzy submodule of the $R$-module $N / N_{j}$ induced by $\nu_{N}$. We claim that the assumption in (ii) implies that:

$$
\gamma_{t}\left(x+N_{t}\right) \neq 1
$$

for all $2 \leq t \leq j$ and non-zero elements $x \in \operatorname{ker}(g)$. Here, $\gamma_{t}$ is the quotient fuzzy submodule of the $R$-module $N / N_{t}$ induced by $\nu_{N}$ and $N_{t}=\sum_{k=1}^{t-1} r_{k} N$. To prove this, let $\gamma_{t}\left(x+N_{t}\right)=1$ for some $x \in \operatorname{ker}(g)$. Then, there exists $y \in N$ such that

$$
x+N_{t}=y+N_{t} \text { and } \nu(y)=1 .
$$

It implies that $x-y \in N_{t} \subseteq N_{j}$ since $t \leq j$. Hence, $x+N_{j}=y+N_{j}$ and $\gamma_{j}\left(x+N_{j}\right)=\nu(y)=1$. By the assumption in $(i i)$, it implies that $x=\theta$. This proves the claim. With the similar arguments, Theorem 5.10, one can prove that $r_{1}, \ldots, r_{j}$ is a fuzzy regular sequence over $\nu_{N}$. 


\section{Conclusions}

Fuzzy sets mathematically represent uncertainty and imprecision. It is a technical tool for dealing with the vagueness inherent in something that is supposed to be precise measurement. It has been successfully applied to many real life problems. The structure of integral domains play an important role in which many of the physical and real world problems are modeled. On the other hand, regular sequences are successfully applied to elimination theory. Elimination theory has an importance in applied and computational mathematics. In this paper, we have given an innovative concept of fuzzy zero-divisors, fuzzy integral domains and fuzzy regular sequences which is the fuzzification of the usual zerodivisors, integral domains and regular sequences in crisp sets. Moreover, some of the interesting and significant results of these notions are proved. We believe that this new idea will be helpful to generate other classical algebraic systems to the fuzzy algebraic systems.

\section{Competing Interests}

The author(s) do not have any competing interests in the manuscript.

\section{Acknowledgement}

The authors are grateful to the reviewers for suggestions to improve the manuscript.

\section{REFERENCES}

1. Zadeh, L. A. (1965). Fuzzy sets. Inform. and Contr., 8, 338-353.

2. Rosenfeld, A. (1971). Fuzzy groups. J. Math. Annal. Appl., 35, 512-517.

3. Asaad, M., \& Abou-Zaid, S. (1996). Characterization of fuzzy subgroups. Fuzzy sets and systems, 77(2), 247-251. https://doi.org/10.1016/0165-0114(95)00234-0

4. Mordeson, J. N. (1994). Invariants of fuzzy subgroups. Fuzzy sets and systems, 63(1), 81-85. https://doi.org/10.1016/0165-0114(94)90147-3

5. Morsi, N. N., \& Yehia, S. E. B. (1994). Fuzzy-quotient groups. Information sciences, 81(3-4), 177-191. https://doi.org/10.1016/0020-0255(94)90096-5

6. Ray, S. (1992). Isomorphic fuzzy groups. Fuzzy Sets and Systems, 50(2), 201-207. https://doi.org/10.1016/0165-0114(92)90219-T

7. Liu, W. J. (1982). Fuzzy invariant subgroups and fuzzy ideals. Fuzzy sets and systems, 8(2), 133-139. https://doi.org/10.1016/0165-0114(82)90003-3

8. Wang-Jin, L. (1983). Operations on fuzzy ideals. Fuzzy sets and systems, 11(1-3), 31-39. https://doi.org/10.1016/S0165-0114(83)80067-0

9. Dixit, V. N., Kumar, R., \& Ajmal, N. (1992). On fuzzy rings. Fuzzy sets and systems, 49(2), 205-213. https://doi.org/10.1016/0165-0114(92)90325-X

10. Kuraoka, T., \& Kuroki, N. (1992). On fuzzy quotient rings induced by fuzzy ideals. Fuzzy sets and Systems, 47(3), 381-386. https://doi.org/10.1016/0165-0114(92)90303-L

11. Mukherjee, T. K., \& Sen, M. K. (1987). On fuzzy ideals of a ring I. Fuzzy sets and systems, 21(1), 99-104. https://doi.org/10.1016/0165-0114(87)90155-2

12. Malik, D. S., \& Mordeson, J. N. (1992). Fuzzy homomorphisms of rings. Fuzzy sets and systems, 46(1), 139-146. https://doi.org/10.1016/0165-0114(92)90275-9 
13. Negoita, C., \& Ralescu, D. (1975). Applications of Fuzzy Sets Systems AnalysisBirkhauser Verlag. Basel und Stuttgart, Ch, 5, 135-142.

14. Pan, F. Z. (1987). Fuzzy finitely generated modules. Fuzzy sets and systems, 21(1), 105113. https://doi.org/10.1016/0165-0114(87)90156-4

15. Pan, F. Z. (1988). Exact sequences of fuzzy linear maps. Fuzzy Sets and Systems, 27(3), 317-325. https://doi.org/10.1016/0165-0114(88)90057-7

16. Pan, F. (1992). The various structures of fuzzy quotient modules. Fuzzy sets and systems, 50(2), 187-192. https://doi.org/10.1016/0165-0114(92)90217-R

17. Muganda, G. C. (1993). Free fuzzy modules and their bases. Information sciences, 72(1-2), 65-82.

18. Kumar, R., Bhambri, S. K., \& Kumar, P. (1995). Fuzzy submodules: some analogues and deviations. Fuzzy Sets and Systems, 70(1), 125-130. https://doi.org/10.1016/01650114(94)00260-E

19. Mordeson, J. N., \& Malik, D. S. (1998). Fuzzy commutative algebra. World scientific.

20. Ajmal, N. (1994). Homomorphism of fuzzy groups, correspondence theorem and fuzzy quotient groups. Fuzzy sets and systems, 61(3), 329-339. https://doi.org/10.1016/01650114(94)90175-9

21. Eisenbud, D. (2013). Commutative Algebra: with a view toward algebraic geometry (Vol. 150). Springer Science \& Business Media.

22. Zahedi, M. M., \& Ameri, R. (1994). Fuzzy exact sequence in category of fuzzy modules. J. Fuzzy Math, 2(2), 409-424.

\section{Saba Ayub}

Department of Mathematics, Quaid-I-Azam University Islamabad, Pakistan. e-mail: sabamianayub@gmail.com

Waqas Mahmood

Department of Mathematics, Quaid-I-Azam University Islamabad, Pakistan.

e-mail: wmahmood@qau.edu.pk 